\title{
The ancient armadillo
}

\author{
SCIENTIFIC NAME \\ Dasypus novemcinctus \\ TAXONOMY \\ PHYLUM: Chordata \\ CLASS: Mammalia \\ ORDER: Cingulata \\ FAMILY: Dasypodidae
}

studies), has a relatively long life span of 10-15 years, tends not to bite, tolerates laboratory procedures and, in the US, is found in large populations. The armadillo has also been bred in captivity for research purposes.

Bacteria prefer the relatively low body temperature of the armadillo $\left(32-35^{\circ} \mathrm{C}\right)$, and armadillos are known to harbor several pathogens including Mycobacterium leprae, which causes the chronic infectious disease leprosy (Hansen's disease). Though often considered a disease of antiquity, leprosy remains an important public health problem throughout the word. If left untreated, leprosy can result in irreversible nerve damage with profound sensory and motor nerve loss, deformity and blindness ${ }^{3}$. Though significant progress has been made in reducing new incidences of the disease, improved diagnostic tests and treatments are still needed ${ }^{4}$.

A major obstacle in leprosy research has been the inability to cultivate $M$. leprae in vitro, making it difficult to produce sufficient quantities of the bacteria for testing. The only animal in which leprosy is reliably recapitulated is the nine-banded armadillo, which exhibits many of the clinical conditions that are associated with the disease in humans ${ }^{3}$.

In laboratories, armadillos are experimentally infected to help find effective vaccines and treatments for leprosy. Armadillos have been used to study both susceptibility and resistance to leprosy. One study with armadillos helped to identify antigens for early leprosy diagnosis, which can be used for screening programs in regions where leprosy is endemic ${ }^{3}$. Now that the Human Genome Consortium has completed the sequencing of the armadillo genome as part of a comparative genomics initiative, the availability of sequence information on the armadillo is likely to rapidly expand the availability of new immunological probes and reagents for use with armadillos and will advance their use as models for leprosy resistance and vaccination and nerve injury ${ }^{5}$.

1. Rosa, P.S., Pinke, C.A.E., Pedrini, S.C.B. \& Silva, E.A. The effect of iron supplementation in the diet of Dasypus novemcinctus (Linnaeus, 1758) armadillos in captivity. Braz. J. Biol. 69, 117-122 (2009).

2. Richini-Pereira, V.B. et al. Importance of xenarthrans in the eco-epidemiology of Paracoccidoides brasiliensis. BMC Res. Notes 2, 228 (2009).

3. Duthie, M.S. et al. Insight toward early diagnosis of leprosy through analysis of the developing antibody responses of Mycobacterium leprae-infected armadillos. Clin. Vaccine Immunol. 18, 254-259 (2011).

4. Pena, M.T. et al. Expression and characterization of recombinant interferon gamma (IFN- $\gamma$ ) from the nine-banded armadillo (Dasypus novemcinctus) and its effect on Mycobacterium leprae-infected macrophages. Cytokine 43, 124-131 (2008).

5. Scollard, D.M. et al. The continuing challenges of leprosy. Clin. Microbiol. Rev. 19, 338 (2006). 